\title{
ARTICLE
}

\section{Peptoids for Biomaterials Science}

Cite this: DOI: 10.1039/x0xx00000x

Received 00th January 2012,

Accepted 00th January 2012

DOI: $10.1039 / \times 0 \times x 00000 x$

www.rsc.org/

\author{
King Hang Aaron Lau* ${ }^{a}$
}

Poly( $N$-substituted glycine) "peptoids" have conventionally been exploited for drug discovery and therapeutics due to their structural similarity to peptides, protease resistance, and relative ease of synthesis. This mini-review highlights recent reports of peptoid selfassembled nanostructures and macromolecular interfaces relevant to biomaterials science. The results illustrate how the versatility of peptoid design and synthesis could be exploited to generate multifunctional, modular and precisely tunable biointerfaces and biomaterials.

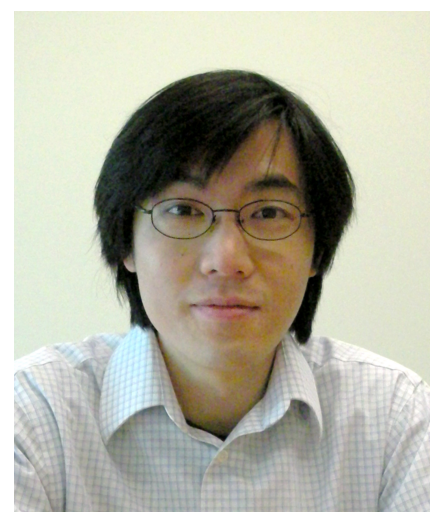

Dr K. H. Aaron Lau is a lecturer in the Department of Pure and Applied Chemistry at the University of Strathclyde. He received his Sc.B. and Sc.M. from Brown University and completed his Ph.D. at the Max Planck Institute for Polymer Research. He was previously a US National Institutes of Health National Research Service Award postdoctoral fellow with Prof Phillip B. Messersmith at Northwestern University. Aaron's core interests are in bioinspired materials and biointerfaces, and he currently directs projects in peptoid biomaterials and bioinspired nanopore applications.

\section{Introduction}

Materials that can be conveniently tuned to present the biochemical, morphological and mechanical features of native biological systems are of special interest to biomaterials science. ${ }^{1,2,3}$ The incorporation of targeting elements minimizes the potential of negative systemic effects, an important translational consideration. ${ }^{1}$ These ideas have inspired significant research in peptidic biomaterials ${ }^{3,4}$ as well as the chemical functionalization of naturally-derived and synthetic polymers. ${ }^{2,5}$ Poly $(N$-substituted glycine) "peptoids" (Figure
1A), a class of highly customizable peptidomimetic macromolecules, could also potentially enable significant advances in biomaterials science.

Peptoids $^{\dagger}$ were developed in the late 1980s for combinatorial drug discovery as synthetically convenient, modular, peptidomimetic molecules. ${ }^{6,7}$ They are structural isomers of peptides with sidechains connected to the amide nitrogens instead of the $\alpha$-carbons (Figure 1A). This structural change confers resistance to protease degradation..$^{7-9}$ The sidechain shift results in tertiary amide linkages, leading to a backbone that is achiral and that has no H-bond donors. These properties confer substantial conformational flexibility in the main chain. ${ }^{10-14}$ Chirality and control over secondary and higher order structure formation can be reintroduced by sidechain chemistry and sequence designs..$^{10,12,13,15}$

Synthetic convenience and modularity in generating sequences with monomer-level programmability over sidechain chemistry and chain length derive largely from the introduction of the submonomer solid phase synthesis by Zuckermann et al. (Figure 1B). ${ }^{7,16}$ No backbone protection is required and diverse primary amines, many of which are commercially available, can be incorporated as sidechains in an iterative protocol. Over 250 different residues, including analogs or close mimics of all the canonical amino acids, have been demonstrated. ${ }^{17,18}$ Peptoids of up to 100 residues in length have been demonstrated by coupling together two submonomersynthesized 50-mers. ${ }^{19}$ Alternatively peptoids can be synthesized with high degrees of polymerization $>100,{ }^{20}$ but with limitations in sequence control, by the living polymerization of $\mathrm{N}$-carboxy anhydride (NCA) monomers 
(Figure 1C). ${ }^{21-23}$ The polymer of sarcosine, which is the analog of alanine and the simplest peptoid, was actually known from early investigations into living polymerization. ${ }^{21}$ The list of peptoid monomers available to NCA polymerization has been significantly expanded in recent years. ${ }^{21,22}$

The biorecognition abilities of peptoids have been demonstrated through two decades of peptoid therapeutics research, protein-binding sequence discovery, and secondary structure design, which have been recently reviewed elsewhere. ${ }^{10,15,24-27}$ As originally envisioned, ${ }^{7}$ many bioactive sequences have been discovered by screening peptoid libraries. $^{8,13}$ These include antimicrobial peptoids - a significant area of peptoid research discussed in recent reviews. ${ }^{10,25,27}$ Recent studies have also demonstrated peptoid sequences potentially useful in diagnosing or treating amyloid diseases. ${ }^{28}$

This mini-review highlights the recent developments in peptoids research which extend into the field of biomaterials science. A number of recent reviews have focused on how the synthetic accessibility and sequence programmability in chemistry and chain length of peptoids have generated broad potential in macromolecular and nanostructure engineering. $^{12,13,21,22}$ This mini-review first discusses the biomaterial applications of these self-assembled structures. Attention is then turned to peptoid macromolecular interfaces and platforms that could offer unique possibilities. The aim is to illustrate how the versatility in peptoid design and synthesis can lead to macromolecular architectures with high potential in biomaterials science.
A)

$$
\text { peptoid }
$$

B)<smiles>[R]N(C)CC(=O)N([R])CC(=O)NCC(C)=O</smiles>

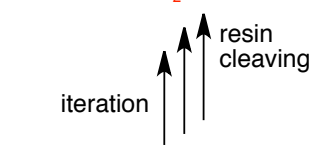

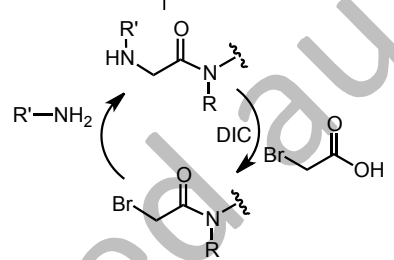

C)

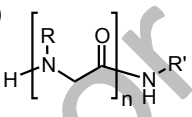
$\uparrow-\mathrm{nCO}_{2}$ $\mathrm{H}_{2} \mathrm{~N}-\mathrm{R}^{\prime}$ $(\underbrace{n} \underbrace{0}$
Figure 1. Peptoid chemical structure and synthesis. A) A shift in the sidechain connection from the $\alpha$-carbon to the amide nitrogen, accompanied by loss of main chain chirality and amide hydrogens, differentiates peptoids from peptides (Lamino acid residue shown). B) Submonomer solid phase synthesis: residue coupling is split into elongating the chain via acylation with a haloacetic acid and sidechain introduction via displacement with a primary amine submonomer. DIC: $N, N^{\prime}-$ Diisopropylcarbodiimide. C) NCA peptoid polymerization.

\section{Self-Assembled Systems}

\subsection{Molecular (Hydro)gelators}

A pair of studies by $\mathrm{Wu}$ et al. ${ }^{29}$ and Mangunuru et al. ${ }^{30}$ demonstrate that low molecular weight peptoid sequences could be utilized as gelators. Wu et al. picked a sequence of four peptoid analogs of phenylalanine (Phe) as the gelation motif (Figure 2; peptoid phenylalanine is shortened to NPhe below). The tetrapeptoid (NPhe) ${ }_{4}$ was linked via a glycine residue to tripeptides with demonstrated bioactivity (RGD, YSV and VPP), as well as a triglycine GGG control. Gels of all four sequences could be formed in phosphate buffered saline at a concentration of $10 \mathrm{mg} / \mathrm{mL}$ at $\mathrm{pH} 7.4$. However the behavior of standalone (NPhe $)_{4}$ and $(\mathrm{NPhe})_{4} \mathrm{G}$ were not reported. The hydrogels were composed of either nanosheets or nanofibrils (Figure 2A-C), and the highest shear storage modulus was observed for (NPhe) ${ }_{4}$ GGGG (750 Pa over $0.1-100 \mathrm{rad} / \mathrm{s}$ ). The viabilities of several cell lines in solutions of the peptoids were not seriously affected (tested up to $200 \mu \mathrm{M}$ ). Fibroblasts also cultured well on the (NPhe) ${ }_{4}$ GRGD and, especially, on the (NPhe) $)_{4}$ GYSV gels. Finally, it was demonstrated that the (NPhe) $)_{4}$ sequence, but not (D-Phe $)_{4}$ nor (L-Phe) $)_{4}$, was effective in protecting the pendant RGD sequences from degradation by proteinase $\mathrm{K}$.

In a different approach, Mangunuru et al. scanned for gelators among a small library synthesized by Ugi one-pot fourcomponent reactions. ${ }^{30}$ Single peptoid residues functionalized at both the $\mathrm{C}$ and $\mathrm{N}$ "termini" were generated. Many of the sidechain functional groups investigated have previously been demonstrated using the submonomer solid phase protocol. ${ }^{17}$ Although Mangunuru et al. reported only gels formed in water mixed with $\geq 33 \%$ ethanol or dimethyl sulfoxide (DMSO), this work highlighted the chemical tunability possible with peptoids and the chemical groups that could promote peptoid gelation.

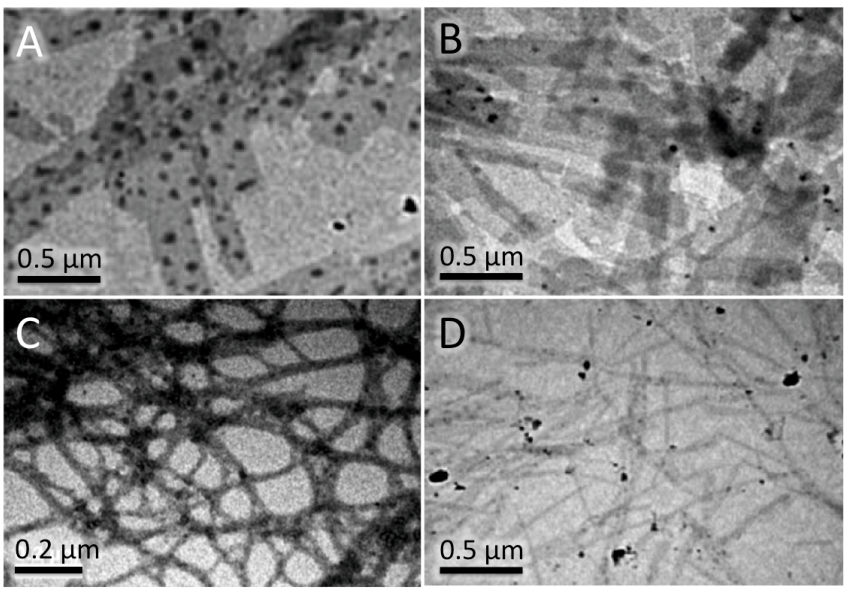

Figure 2. Peptoid hydrogels. TEM images of hydrogels composed of A) (NPhe) ${ }_{4} \mathrm{GGGG}, \mathrm{B}$ ) (NPhe) $\left.{ }_{4} \mathrm{GRGD}, \mathrm{C}\right)(\mathrm{NPhe})_{4} \mathrm{GYSV}$, and D) (NPhe) ${ }_{4}$ GVPP. The first two hydrogels were composed of sheet-like microstructures while the latter two were formed from filamentous structures. Adapted from Ref. 29 with permission from The Royal Society of Chemistry.

The best gelators reported by Mangunuru et al. exhibited substituted aryl, cyclohexyl or protected glucosamine groups, while combinations with unsubstituted benzyl groups did not gel well. This is in apparent contrast to the gelation of the (NPhe) $)_{4}$ hybrids reported by $\mathrm{Wu}$ et al. However, the multiple 
unsubstituted benzyls in the (NPhe) $)_{4}$ motif could induce a larger hydrophobic interaction between the peptoids. Juxtaposition of (NPhe) 4 with the hydrophilic peptide sequences could also induce amphiphile self-assembly. In fact, the combination of hydrophobic and amphiphile interactions feature prominently in the self-assembly of many peptoid nanostructures, as described below.

\subsection{Nano and Micro Structures}

NANOSHEETS which are two molecular layers thick and water soluble have recently been applied as an antibody-mimetic platform by Zuckermann et al. (Figure 3). ${ }^{31}$ The researchers had earlier found that nanosheets can be assembled at the air-water interface from sequences that laterally align to form two opposing layers (Figure 3B). ${ }^{32,33}$ Assembly required the electrostatic interaction between oppositely charged peptoid residues, and the alternation of hydrophilic and hydrophobic residues such that a hydrophobic core can be formed between the opposing layers (Figure 3A-C). Overall sequence lengths of 12 or more residues were required to build up a sufficiently attractive interaction. Zuckermann et al. then inserted oligopeptide segments with known recognition properties between peptoid sequences possessing the periodic selfassembly motif (Figure 3D). These sequences also assembled into nanosheets, with the oligopeptides "squeezed out" as dangling loops. The nanosheets thus acted as a structural scaffold for the presentation of biorecognition loops and mimicked the conceptual structure of antibodies (Figure 3E).

Molecular recognition was demonstrated by three examples: i) the serine of a consensus peptide of casein kinase II presented on the nanosheets could be phosphorylated by the enzyme; ii) proteases were able to digest the presented peptides without disrupting the nanosheets; and iii) gold nanocrystals could be grown from E. coli gold-binding peptides presented on the nanosheets. The peptoid nanosheets do not aggregate in buffer, possibly due to the zwitterionic nature of the nanosheet surface (Figure 3B). There is no critical peptoid concentration once the nanosheets have been assembled but the sheets can be designed to re-dissolve at a certain $\mathrm{pH}$ by tuning the sequence arrangement. ${ }^{33}$ Being $2 \mathrm{D}$ objects, the nanosheets possess a high surface area to mass ratio, and could provide a high degree of cargo loading using either the hydrophobic core or a functionalized surface. Together with the commercial availability of the peptoid submonomer precursors used in the self-assembly motif and the modularity of the sequence design, the nanosheets hold significant promise for a range of delivery and sensing applications.

NANO AND MICRO FIBRIL self-assembly have been demonstrated for peptoids synthesized by both the submonomer solid phase and NCA polymerization routes. The Zhang group investigated $^{34}$ NCA diblock copolypeptoids composed of blocks with methylene (i.e. sarcosine-hydrophilic ${ }^{21,35}$ ) and hydrophobic decyl sidechains. These block copolymers were found to self-assemble into spherical micelles that, over the course of several days, transitioned into cylindrical micelles with core diameters of $12 \mathrm{~nm} .{ }^{34}$ Zuckermann et al. found solid phase synthesized amphiphilic diblock sequences that could self-assemble, first into nanosheets that then rolled (also over the course of several days), into microfibrillar helices. ${ }^{36}$ The ability to dial in specific block lengths and arrangements with the submonomer protocol was used to show that self-assembly required both hydrophobic and ionic interactions of amphiphilic sequences.

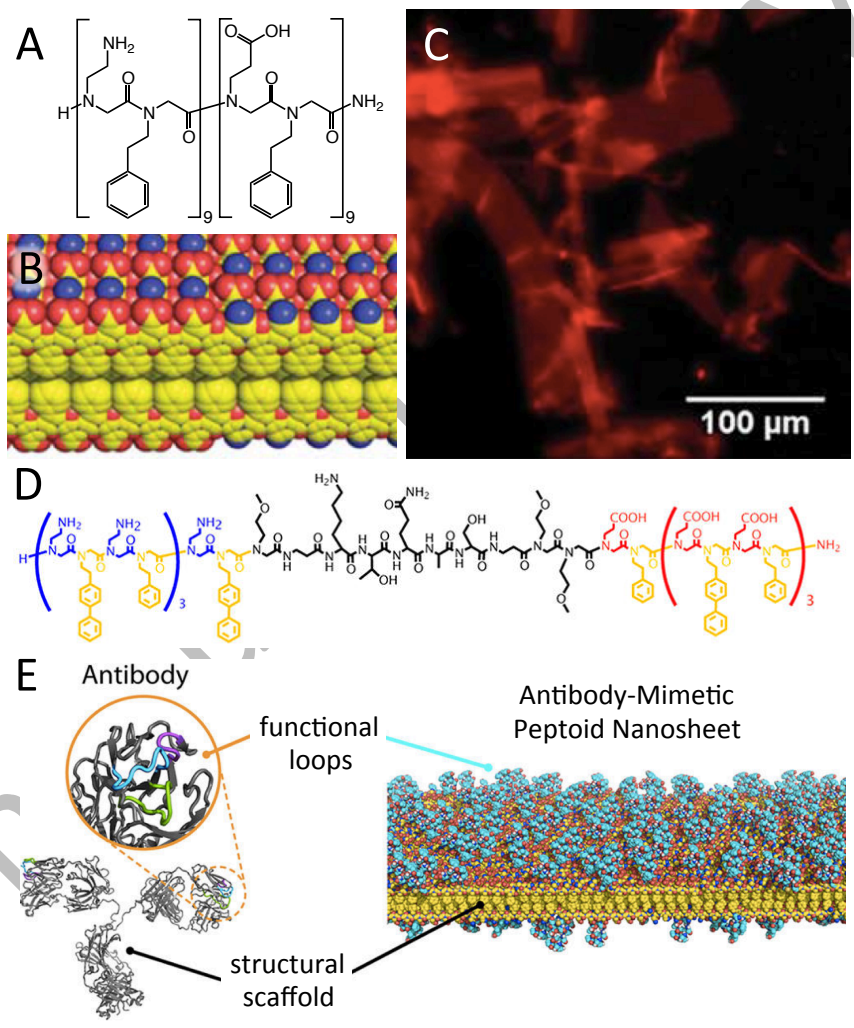

Figure 3. Peptoid nanosheets. A) Chemical structure of a model sequence that can self-assemble into nanosheets, and B) partial view of the nanosheet molecular model showing the sequestration of hydrophobic phenyl-terminated sidechains and the outward presentation of the amine- and carboxylic acid-terminated sidechains (red: oxygen; blue: nitrogen; yellow: carbon). C) Free-floating sheets imaged by fluorescence optical microscopy, stained by Nile Red that had sequestered into the hydrophobic interior of the bilayers. D) Chemical structure of a sequence that formed antibody-mimetic nanosheets, as illustrated in (E). Reprinted with permission from Ref. 31 (Copyright 2013 American Chemical Society) and from Ref. 33 (Copyright 2011 Wiley Periodicals).

NANOPARTICLES have been associated with the earliest peptoid research. Sequences with repeating triplet cationichydrophobic-hydrophobic motifs were found to form nanoparticulate DNA-peptoid complexes that were efficient gene transfection agents. ${ }^{37}$ Similar sequences, as well as polyamphoteric ones, have recently been shown to form microspheres. ${ }^{38}$ In a different line of research, hydrophilic polysarcosine synthesized by NCA polymerization have been exploited for controlling the water solubility of amphiphilic peptoid-peptide/polymer block copolymers that self-assemble into (nano)particles for controlled release and imaging 
applications. ${ }^{39}$

\section{Macromolecular Interfaces and Platforms}

\subsection{Antifouling Peptoids}

Peptoid chains grafted on solid surfaces as polymer brushes have been demonstrated to confer excellent resistances against protein adsorption and cell attachment. ${ }^{35,40-43}$ Several peptoid sidechains and chain lengths have been investigated. Intriguingly, polymer brushes composed of sarcosine, the peptoid with the simplest sidechain (a single methylene: Figure 4), exhibited antifouling performance that is similarly excellent as peptoids with methoxyethyl sidechains that resemble the repeating unit of PEG. ${ }^{35,40,42}$ In both cases, protein adsorption could essentially be prevented at chain lengths of just 20 repeating units, and long term fibroblast attachment could also be prevented (Figure 4). ${ }^{35,40}$ The resistance against bacteria attachment ranged from $75 \%$ to $99 \%$ ( $1 \mathrm{~d}$ ), depending on the combination of the sidechain and the bacteria strain being tested. $^{35,41}$

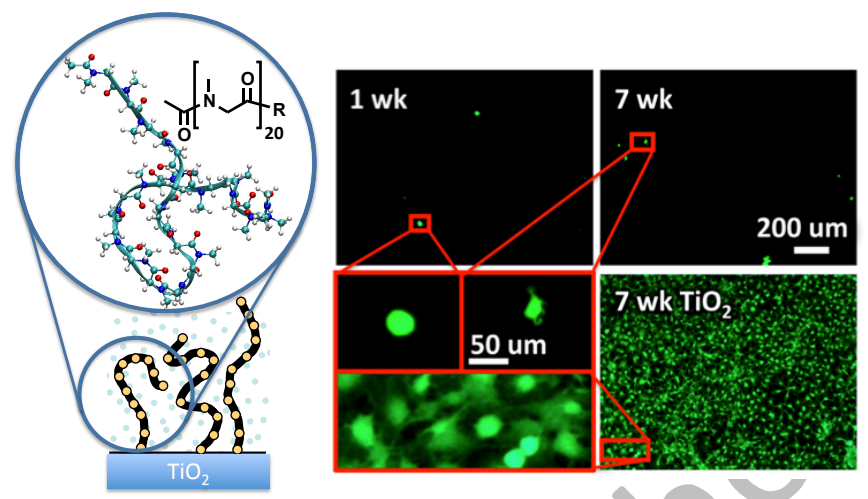

Figure 4. Antifouling polysarcosine surface grafted peptoid brush. The left shows the schematic, chemical structure and molecular dynamics snapshot of the polysarcosine 20-mer used in antifouling experiments. The fluorescence micrographs on the right show that the number of 3T3 mouse fibroblasts attached on the polysarcosine-coated surface was very low through $7 \mathrm{wk}$, while a confluent layer was achieved on the uncoated $\mathrm{TiO}_{2}$ control surface during the same period (only $7 \mathrm{wk}$ data shown). Fresh fibroblasts were reseeded on the samples twice a week. Although some cells were observed on the polysarcosine surface, their morphologies were rounded, exhibiting few filopodia, and were poorly attached. Reprinted with permission from Ref. 35. Copyright 2012 American Chemical Society.

According to molecular theory, the chain length and grafting density of a well-solubilized, flexible polymer brush dominate its antifouling performance. ${ }^{42,44}$ As such the short persistence length and inherent flexibility of the peptoid backbone, ${ }^{11}$ conferred by its achirality and the isomerization between trans and cis conformations, ${ }^{14}$ render hydrophilic peptoids an attractive antifouling platform. Consistent with this observation, excellent antifouling performance was also shown for $\beta$-peptoids with methyl and ethyl sidechains. ${ }^{45}$ Last but not least, the flexibility in peptoid synthesis enables convenient functionalization of antifouling peptoid biointerfaces, as demonstrated by peptoid brushes decorated with saccharides ${ }^{46}$ and antimicrobial peptides. ${ }^{41}$

\subsection{Clickable Multivalent Scaffolds}

Using the submonomer protocol, Kirshenbaum et al. demonstrated that azide-alkyne cycloaddition on alkyne- or azide-terminated sidechains can be used to attach, in a sequence-specific manner, multiple and multifarious functional groups that are otherwise difficult to incorporate. ${ }^{47}$ These included estradiol, a hormone, and the electrochemically active ethynylferrocene. Polysaccharide mimics that can efficiently bind Concanavalin A have been demonstrated by clicking on mannose sidechains to a peptoid scaffold. ${ }^{48}$ The easy and precise chain length control by submonomer peptoid synthesis was exploited to determine that efficient binding to Concanavalin A required peptoid-mannose sequences of five or more residues. Similarly, control in the attachment positions of aminoglycoside sidechains clicked onto a peptoid backbone, separated by "spacer" residues with propyl sidechains, was used to tailor the specificity in the aminoglycoside targeting of RNAs that cause myotonic muscular dystrophy. ${ }^{49}$ Furthermore polyglycerated and polyglycosylated peptoids have been demonstrated by thiol-ene click addition to a NCA polymerized peptoid with allyl sidechains. ${ }^{50}$ Peptoid macrocycles with alkyne-terminated sidechains have also been synthesized by both the submonomer protocol ${ }^{51}$ and NCA polymerization. ${ }^{52}$ Depending on the sequence, the macrocycles could crystallize and adopt two interconvertable conformations and reversibly sequester water. ${ }^{51}$

\subsection{Peptoid Libraries for Materials Discovery.}

Peptoid combinatorial libraries have been fruitfully used for therapeutics and protein-binding sequence discovery (see Introduction). Recent reports highlight the utility of the approach for materials applications. The concept has been applied to the discovery of small molecule gelators from a small library (see section 2.1). Kodadek et al. has developed ${ }^{53}$ a cell-based assay for screening vascular endothelial growth factor receptor 2 (VEGFR2)-binding peptoids that could potentially be adapted for biomaterials discovery. Cells expressing VEGFR2 were fluorescently labelled and exposed to PEG-co-PS solid phase synthesis resin beads $(140-170 \mu \mathrm{m}$ in diameter), each displaying one of over 250,000 possible peptoid 9-mers generated by split-pool combinatorial synthesis from 8 different peptoid residues in 8 reaction vessels. Beads on which VEGFR2-expressing cells attached were selected for automated Edman sequencing of the "hit" peptoids for further affinity refinement. In effect, each bead acted as a test well in a multiwell plate cell attachment assay for parallel evaluation of cell behavior.

In the area of (bio)mineralization, library investigation of amphiphilic peptoids with specific sequences of hydrophobic and acidic sidechains discovered sequences that could accelerate calcite crystal growth by 23 -fold at only $50 \mathrm{nM}$ peptoid concentration (compared with enhancement factors of 
1.64 or less by acidic peptides of similar molecular weights). ${ }^{54}$ In a different study, trimers of a hydroxylated peptoid residue (analog of serine) were discovered to exhibit dual-action antifreeze effects - enhanced ice growth inhibition and melting temperature reduction. ${ }^{55}$ Both studies found that the sequence of peptoid additives could be used to control the crystal morphology, and that specific sequences likely altered the interactions of water molecules with the crystallizing surfaces. These reports also illustrate that libraries of sequence-specific peptoids enable the mechanistic investigation of how variations in chemical structure alter performance.

\section{Summary and Outlook}

The studies reviewed indicate the wide range of potential biomaterial and biointerfacial applications enabled by peptoids. Peptoid hydrogelators that are compatible with cell culture have been found. The modular design of self-assembled peptoid nanosheets with bioactive sequences has been demonstrated, opening up potential applications in drug delivery and biosensing. Peptoid nano and micro fibrils can also be selfassembled, and peptoid nanoparticles have been explored for gene transfection and controlled release applications. Biointerfaces in the guise of antifouling peptoid polymer brushes that can be further functionalized with saccharides and antimicrobial peptides have been demonstrated. Clickable multivalent peptoid scaffolds lower the synthetic barrier to the introduction of complex sidechains and polysaccharide mimics. In addition, peptoid libraries hold significant promise for biomaterials discovery.

The knowledge gained from two decades of peptoid peptidomimetics research ${ }^{10,13,24-26,56}$ could potentially be quickly translated for biomaterials applications. Design rules for dialing in secondary structure and peptoid folding by sidechain design and sequence control have emerged. ${ }^{10,13,15} \mathrm{~A}$ catalogue of bioactive peptoids has been included in a recent review. ${ }^{17}$ Peptoids have also been shown to exhibit enhanced cell penetration compared to the corresponding peptide sequences, ${ }^{57}$ but they can also be tuned, as in the case of some antimicrobial peptoids, to target bacterial membranes. ${ }^{58}$ Although the increased conformational flexibility of the peptoid backbone is expected to decrease binding affinity ${ }^{24}$ (e.g. integrin receptor binding by direct peptoid analogs of RGD was either not reported ${ }^{59}$ or not elicited ${ }^{60}$ ), certain peptoid sequences lacking secondary structure appear to possess activities superior to their structured counterparts. ${ }^{10,58,61}$ The resistance of peptoids against protease degradation ${ }^{8,9,18,31,41}$ is also potentially beneficial for protecting a pharmacological cargo, ${ }^{37}$ enhancing stability and bioavailability, ${ }^{6,7,24}$ and long-term biomedical applications, such as antifouling coatings. ${ }^{40}$

A continuing catalyst for peptoids research is the relative synthetic ease with which the sidechain chemistry and sequence can be precisely tuned to achieve the desired property. Stimuliresponsiveness encoded by the sequence design would undoubtedly be advantageous, and $\mathrm{pH}^{33}$ temperature $^{62}$ and solvent condition ${ }^{19}$ effects have been reported. Peptoids could be used to mediate nanoparticle assembly ${ }^{63}$ and to enhance the stability of nanoparticles in difficult ionic conditions. ${ }^{64}$ Biodegradability could potentially be reintroduced by inserting suitable peptide sequences. An interesting challenge remains in the routine synthesis of long, sequence-specific peptoids (e.g. > 100 residues, as in many protein amino acid sequences). Ultimately, a bright future awaits the application of peptoids in biomaterials science as researchers further exploit the knowledge gained through peptoid therapeutics research and the highly accessible chemical versatility, self-assembly capabilities, and other intrinsic properties of peptoids.

\section{Acknowledgements}

KHAL acknowledges support from the University of Strathclyde Strategic Academic Investment Scheme (SAIS).

\section{Notes and references}

${ }^{a}$ Department of Pure and Applied Chemistry, University of Strathclyde, Thomas Graham Building, 295 Cathedral Street, Glasgow, G1 1XL, U.K.

E-mail: aaron.lau@strath.ac.uk; Tel: +44 (0) 1415482162

${ }^{\dagger}$ Although the term "peptoids" was originally conceived ${ }^{65}$ as any nonpeptidic chemical analog that mimics the biological actions of peptides, and has been used to describe a number of different peptidomimetic polyamides, most reports of peptoids, e.g. those indexed in PubMed, refer to $N$-substituted glycines.

1. E. T. Pashuck and M. M. Stevens, Sci. Transl. Med., 2012, 4.

2. R. A. Petros and J. M. DeSimone, Nature Reviews Drug Discovery, 2010, 9, 615; O. Z. Fisher, A. Khademhosseini, R. Langer and N. A. Peppas, Acc. Chem. Res., 2010, 43, 419.

3. D. Sengupta and S. C. Heilshorn, Tissue Eng. Part B-Rev., 2010, 16, 285; J. P. Jung, J. Z. Gasiorowski and J. H. Collier, Biopolymers, 2010, 94, 49.

4. S. L. Kuan, Y. Z. Wu and T. Weil, Macromol. Rapid Commun., 2013, 34, 380; R. L. DiMarco and S. C. Heilshorn, Adv. Mater., 2012, 24, 3923; M. Zelzer and R. V. Ulijn, Chem. Soc. Rev., 2010, 39, 3351.

5. M. Malmsten, Current Opinion in Colloid \& Interface Science, 2013, 18, 468; O. F. Khan and M. V. Sefton, Trends Biotechnol., 2011, 29, 379.

6. R. J. Simon, R. S. Kania, R. N. Zuckermann, V. D. Huebner, D. A. Jewell, S. Banville, S. Ng, L. Wang, S. Rosenberg, C. K. Marlowe, D. C. Spellmeyer, R. Y. Tan, A. D. Frankel, D. V. Santi, F. E. Cohen and P. A. Bartlett, Proc. Natl. Acad. Sci. U. S. A., 1992, 89, 9367.

7. R. N. Zuckermann, Biopolymers, 2011, 96, 545.

8. S. M. Miller, R. J. Simon, S. Ng, R. N. Zuckermann, J. M. Kerr and W. H. Moos, Bioorg. Med. Chem. Lett., 1994, 4, 2657.

9. S. M. Miller, R. J. Simon, S. Ng, R. N. Zuckermann, J. M. Kerr and W. H. Moos, Drug Dev. Res., 1995, 35, 20.

10. S. A. Fowler and H. E. Blackwell, Organic \& Biomolecular Chemistry, 2009, 7, 1508.

11. A. M. Rosales, H. K. Murnen, S. R. Kline, R. N. Zuckermann and R. A. Segalman, Soft Matter, 2012, 8, 3673. 
12. A. M. Rosales, R. A. Segalman and R. N. Zuckermann, Soft Matter, 2013, 9, 8400

13. J. Sun and R. N. Zuckermann, ACS Nano, 2013, 7, 4715.

14. K. Kirshenbaum, A. E. Barron, R. A. Goldsmith, P. Armand, E. K. Bradley, K. T. V. Truong, K. A. Dill, F. E. Cohen and R. N. Zuckermann, Proc. Natl. Acad. Sci. U. S. A., 1998, 95, 4303; Q. Sui, D. Borchardt and D. L. Rabenstein, J. Am. Chem. Soc., 2007, 129, 12042

15. B. Yoo and K. Kirshenbaum, Curr. Opin. Chem. Biol., 2008, 12, 714; A. M. Czyzewski and A. E. Barron, AICHE J., 2008, 54, 2.

16. R. N. Zuckermann, J. M. Kerr, S. B. H. Kent and W. H. Moos, J. Am. Chem. Soc., 1992, 114, 10646.

17. A. S. Culf and R. J. Ouellette, Molecules, 2010, 15, 5282.

18. J. A. W. Kruijtzer, L. J. F. Hofmeyer, W. Heerma, C. Versluis and R. M. J. Liskamp, Chemistry - A European Journal, 1998, 4, 1570 .

19. H. K. Murnen, A. R. Khokhlov, P. G. Khalatur, R. A. Segalman and R. N. Zuckermann, Macromolecules, 2012, 45, 5229.

20. M. Schneider, C. Fetsch, I. Amin, R. Jordan and R. Luxenhofer, Langmuir, 2013, 29, 6983; N. Gangloff, C. Fetsch and R. Luxenhofer, Macromol. Rapid Commun., 2013, 34, 997.

21. R. Luxenhofer, C. Fetsch and A. Grossmann, J. Polym. Sci. Pol. Chem., 2013, 51, 2731.

22. D. H. Zhang, S. H. Lahasky, L. Guo, C. U. Lee and M. Lavan, Macromolecules, 2012, 45, 5833.

23. H. R. Kricheldorf, Angew. Chem.-Int. Edit., 2006, 45, 5752.

24. R. M. J. Liskamp, D. T. S. Rijkers, J. A. W. Kruijtzer and J. Kemmink, ChemBioChem, 2011, 12, 1626.

25. M. T. Dohm, R. Kapoor and A. E. Barron, Curr. Pharm. Des., 2011 , 17, 2732.

26. T. Kodadek, Curr. Opin. Chem. Biol., 2010, 14, 713.

27. S. Lohan and G. S. Bisht, Mini-Rev. Med. Chem., 2013, 13, 1073.

28. Y. Luo, S. Vali, S. Y. Sun, X. S. Chen, X. Liang, T. Drozhzhina, E. Popugaeva and I. Bezprozvanny, ACS Chem. Neurosci., 2013, 4, 952; A. Y. Yam, X. Wang, C. M. Gao, M. D. Connolly, R. N. Zuckermann, T. Bleu, J. Hall, J. P. Fedynyshyn, S. Allauzen, D. Peretz and C. M. Salisbury, Biochemistry, 2011, 50, 4322; M. M. Reddy, R. Wilson, J. Wilson, S. Connell, A. Gocke, L. Hynan, D. German and T. Kodadek, Cell, 2011, 144, 132; C. M. Gao, A. Y. Yam, X. Wang, E. Magdangal, C. Salisbury, D. Peretz, R. N. Zuckermann, M. D. Connolly, O. Hansson, L. Minthon, H. Zetterberg, K. Blennow, J. P. Fedynyshyn and S. Allauzen, PLoS One, 2010, 5, e15725; R. C. Elgersma, G. E. Mulder, J. A. W. Kruijtzer, G. Posthuma, D. T. S. Rijkers and R. M. J. Liskamp, Bioorg. Med. Chem. Lett., 2007, 17, 1837.

29. Z. D. Wu, M. Tan, X. M. Chen, Z. M. Yang and L. Wang, Nanoscale, 2012, 4, 3644

30. H. P. R. Mangunuru, H. Yang and G. J. Wang, Chem. Commun., 2013, 49, 4489 .

31. G. K. Olivier, A. Cho, B. Sanii, M. D. Connolly, H. Tran and R. N. Zuckermann, ACS Nano, 2013, 7, 9276.

32. K. T. Nam, S. A. Shelby, P. H. Choi, A. B. Marciel, R. Chen, L. Tan, T. K. Chu, R. A. Mesch, B. C. Lee, M. D. Connolly, C. Kisielowski and R. N. Zuckermann, Nat. Mater., 2010, 9, 454; B. Sanii, R. Kudirka, A. Cho, N. Venkateswaran, G. K.
Olivier, A. M. Olson, H. Tran, R. M. Harada, L. Tan and R. N. Zuckermann, J. Am. Chem. Soc., 2011, 133, 20808.

33. R. Kudirka, H. Tran, B. Sanii, K. T. Nam, P. H. Choi, N. Venkateswaran, R. Chen, S. Whitelam and R. N. Zuckermann, Biopolymers, 2011, 96, 586.

34. C. U. Lee, T. P. Smart, L. Guo, T. H. Epps and D. H. Zhang, Macromolecules, 2011, 44, 9574.

35. K. H. A. Lau, C. Ren, T. S. Sileika, S. H. Park, I. Szleifer and P. B Messersmith, Langmuir, 2012, 28, 16099.

36. H. K. Murnen, A. M. Rosales, J. N. Jaworski, R. A. Segalman and R. N. Zuckermann, J. Am. Chem. Soc., 2010, 132, 16112.

37. J. E. Murphy, T. Uno, J. D. Hamer, F. E. Cohen, V. Dwarki and R. N. Zuckermann, Proc. Natl. Acad. Sci. USA, 1998, 95, 1517.

38. M. L. Hebert, D. S. Shah, P. Blake, J. P. Turner and S. L. Servoss, Organic \& Biomolecular Chemistry, 2013, 11, 4459.

39. S. Kimura, T. Kidchob and Y. Imanishi, Polym. Adv. Technol., 2001, 12, 85; A. Makino, E. Hara, I. Hara, R. Yamahara, K. Kurihara, E. Ozeki, F. Yamamoto and S. Kimura, J. Controlled Release, 2012, 161, 821.

40. A. R. Statz, R. J. Meagher, A. E. Barron and P. B. Messersmith, $J$. Am. Chem. Soc., 2005, 127, 7972.

41. A. R. Statz, J. P. Park, N. P. Chongsiriwatana, A. E. Barron and P. B. Messersmith, Biofouling, 2008, 24, 439.

42. K. H. A. Lau, C. Ren, S. H. Park, I. Szleifer and P. B. Messersmith, Langmuir, 2012, 28, 2288.

43. A. R. Statz, J. H. Kuang, C. L. Ren, A. E. Barron, I. Szleifer and P. B. Messersmith, Biointerphases, 2009, 4, FA22.

44. J. Satulovsky, M. A. Carignano and I. Szleifer, Proc. Natl. Acad. Sci. USA, 2000, 97, 9037.

45. S. H. Lin, B. Zhang, M. J. Skoumal, B. Ramunno, X. P. Li, C. Wesdemiotis, L. Y. Liu and L. Jia, Biomacromolecules, 2011, 12, 2573.

46. H. O. Ham, S. H. Park, J. W. Kurutz, I. G. Szleifer and P. B. Messersmith, J. Am. Chem. Soc., 2013, 135, 13015.

47. J. M. Holub, H. J. Jang and K. Kirshenbaum, Organic \& Biomolecular Chemistry, 2006, 4, 1497.

48. H. Yuasa, H. Honma, H. Hashimoto, M. Tsunooka and K. KojimaAikawa, Bioorg. Med. Chem. Lett., 2007, 17, 5274.

49. M. M. Lee, J. L. Childs-Disney, A. Pushechnikov, J. M. French, K. Sobczak, C. A. Thornton and M. D. Disney, J. Am. Chem. Soc., 2009, 131, 17464; M. D. Disney, M. M. Lee, A. Pushechnikov and J. L. Childs-Disney, ChemBioChem, 2010, 11, 375.

50. J. W. Robinson and H. Schlaad, Chem. Commun., 2012, 48, 7835.

51. S. B. L. Vollrath, C. H. Hu, S. Brase and K. Kirshenbaum, Chem. Commun., 2013, 49, 2317.

52. S. H. Lahasky, W. K. Serem, L. Guo, J. C. Garno and D. H. Zhang, Macromolecules, 2011, 44, 9063.

53. D. G. Udugamasooriya, S. P. Dineen, R. A. Brekken and T. Kodadek, J. Am. Chem. Soc., 2008, 130, 5744.

54. C.-L. Chen, J. Qi, R. N. Zuckermann and J. J. DeYoreo, J. Am. Chem. Soc., 2011, 133, 5214.

55. M. L. Huang, D. Ehre, Q. Jiang, C. H. Hu, K. Kirshenbaum and M. D. Ward, Proc. Natl. Acad. Sci. U. S. A., 2012, 109, 19922.

56. C. A. Olsen, ChemBioChem, 2010, 11, 152. 
57. Y.-U. Kwon and T. Kodadek, J. Am. Chem. Soc., 2007, 129, 1508; N. C. Tan, P. Yu, Y. U. Kwon and T. Kodadek, Biorg. Med. Chem., 2008, 16, 5853.

58. N. P. Chongsiriwatana, J. A. Patch, A. M. Czyzewski, M. T. Dohm, A. Ivankin, D. Gidalevitz, R. N. Zuckermann and A. E. Barron, Proc. Natl. Acad. Sci. U. S. A., 2008, 105, 2794.

59. O. E. Vercillo, C. K. Z. Andrade and L. A. Wessjohann, Org. Lett., 2008, 10, 205.

60. I. Dijkgraaf, J. A. W. Kruijtzer, C. Frielink, A. C. Soede, H. W. Hilbers, W. J. G. Oyen, F. H. M. Corstens, R. M. J. Liskamp and O. C. Boerman, Nucl. Med. Biol., 2006, 33, 953.

61. T. Hara, S. R. Durell, M. C. Myers and D. H. Appella, J. Am. Chem. Soc., 2006, 128, 1995.

62. S. H. Lahasky, X. K. Hu and D. H. Zhang, ACS Macro Letters, 2012, 1,580 .

63. G. Maayan and L. K. Liu, Biopolymers, 2011, 96, 679.

64. D. B. Robinson, G. M. Buffleben, M. E. Langham and R. N. Zuckermann, Biopolymers, 2011, 96, 669.

65. P. S. Farmer and E. J. Ariëns, Trends Pharmacol. Sci., 1982, 3, 362. 